\title{
Favoriser la co-régulation et la co-construction d'une démarche efficace de résolution de problèmes mathématiques en fin d'enseignement primaire
}

\section{Doriane Jaegers, Dominique Lafontaine et Annick Fagnant}

La présente étude expérimente un dispositif d'enseignement portant sur des tâches complexes de mathématiques auprès d'élèves de Ge primaire (élèves de 11-12 ans) en Belgique francophone. Ce dernier se base sur les principes constitutifs du modèle d'environnements d'enseignementlapprentissage stimulants (De Corte \& Verschaffel, 2008), ainsi que sur les travaux d'Allal (2007, 2011) et Mottier Lopez (2012) relatifs aux régulations interactives et à la co-régulation. L'étude combine un dispositif quasi-expérimental et des analyses qualitatives. Les résultats mettent en évidence l'efficacité du dispositif développé, y compris auprès des élèves les plus faibles, à condition de fournir un étayage adéquat. Les comparaisons entre classes expérimentale et contrôle soulignent le rôle central de l'enseignant pour faire évoluer le raisonnement des élèves.

\section{Introduction}

Depuis plusieurs années, l'approche par compétences s'est répandue dans les pays francophones (Eurydice, 2012). Avec ses détracteurs et ses adeptes, cette approche a fait couler beaucoup d'encre en Belgique francophone (voir notamment Beckers, 2012; Carette, 2007; Chenu, Crahay \& Lafontaine, 2014; Crahay, 2006; Rey, Carette, Defrance $\&$ Kahn, 2006). Un des piliers constitutifs de celle-ci est la notion de tâche complexe qui, selon plusieurs auteurs, devrait non seulement servir de cadre d'évaluation des compétences, mais aussi de moyen pédagogique pour les acquérir (Rey et al., 2006; Jonnaert, 2009). Dans l'optique d'aider les enseignants à percevoir les tenants et aboutissants de cette approche, des commissions ont développé des «outils d'évaluation des compétences» qui sont aujourd'hui disponibles sur le site du Ministère de l'enseignement de la Fédération Wallonie-Bruxelles (www.enseignement.be). Sans entrer dans le vif débat autour des compétences, le présent article s’intéresse aux tâches complexes de mathématiques à l'école primaire. Ces dernières s'apparentent à des problèmes arithmétiques impliquant plusieurs étapes de résolution et nécessitant d'exploiter 
divers types de documents (horaires, tarifs, plan...). Elles nécessitent la mise en œuvre d'un "processus complexe de modélisation mathématique» (Van Dooren, Verschaffel, Greer, De Bock \& Crahay, 2015; Verschaffel \& De Corte, 2008) au sein duquel deux aspects ont largement retenu l'attention des chercheurs: la construction d'une représentation de la situation (Fagnant \& Vlassis, 2013) et le développement de stratégies métacognitives de contrôle et de vérification (Focant \& Grégoire, 2008; Mevarech \& Kramarski, 2014).

La présente étude expérimente un dispositif d'enseignement/apprentissage portant sur des tâches complexes de mathématiques auprès d'une classe d'élèves de $6^{\mathrm{e}}$ année primaire (grade 6, élèves de 11-12 ans) en Belgique francophone. Ce dispositif a été conçu spécifiquement pour répondre à deux préoccupations majeures. Tout d'abord, de nombreuses recherches (Carette, 2007; Crahay \& Detheux, 2005; Demonty \& Fagnant, 2014; Marcoux, 2014) ont constaté les faibles taux de réussite des élèves face aux tâches complexes. En effet, selon Crahay et Detheux (2005), les élèves seraient capables d'appliquer les procédures lorsqu'elles sont sollicitées directement, mais rencontreraient davantage de difficultés à gérer la complexité des tâches soumises, c'est-à-dire à identifier et mobiliser les procédures adéquates. Ainsi, même quand ils maitrisent les procédures mathématiques, certaines compétences propres à la mise en œuvre d'une démarche efficace de résolution de problèmes sembleraient leur faire défaut. Par ailleurs, des recherches récentes ont soulevé les difficultés rencontrées par les enseignants pour aider les élèves face aux tâches complexes. Procéder à une analyse collective des tâches pour laisser aux élèves la gestion des aspects plus «techniques» (Ginsburg, Cooke, Leinwand \& Pollock, 2005), réaliser un cadrage trop étroit permettant aux élèves de "réussir» sans nécessairement "comprendre" (Bonnery, 2009) ou encore organiser des corrections collectives guidées par la logique de l'adulte (Demonty \& Fagnant, 2014; Fagnant, Dupont \& Demonty, 2016) sont autant d'éléments fréquemment rencontrés, mais dont l'efficacité demeure questionnable.

Afin d'éviter que les tâches complexes s'inscrivant dans une approche par compétences ne s'adressent qu'à une certaine élite, il convient de dégager des pistes d'actions efficaces pour aider les élèves et les enseignants à faire face aux instructions officielles. Dans la perspective d'une recherche collaborative, le dispositif a été construit et expérimenté en interaction avec une enseignante. Il se base sur les principes constitutifs du modèle d'environnements d'enseignement/ apprentissage stimulants tel que défini par De Corte, Verschaffel et Masui (2004; voir aussi De Corte \& Verschaffel, 2008), ainsi que sur les travaux d'Allal et Mottier Lopez relatifs aux régulations interactives et à la co-régulation (Allal, 2007, 2011; Mottier Lopez, 2012). Combinant dispositif quasi-expérimental (prétest, post-test, classes expérimentale et contrôle) et analyses qualitatives des interactions observées dans les classes, la présente étude propose une approche mixte qui, sans prétendre à une quelconque généralisation des résultats, tente de tirer parti de recherches issues de divers champs théoriques en vue de développer 
une approche d'enseignement/apprentissage potentiellement porteuse du développement de compétences en résolution de problèmes mathématiques.

\section{Fondements théoriques}

Selon De Corte et Verschaffel (2008), un apprentissage des mathématiques efficace relève d'un "processus constructif, autorégulé, contextualisé et collaboratif d'acquisition de connaissances et d'habiletés» (p. 38). Ces auteurs ont ainsi développé un modèle d'environnements d'enseignement/apprentissage stimulants basé sur cinq principes: (1) susciter des processus d'acquisition actifs et constructifs de la part de tous les élèves; (2) encourager le développement de stratégies d'autorégulation; (3) proposer des situations authentiques ayant une signification personnelle pour l'apprenant; (4) permettre aux élèves de développer les connaissances spécifiques au domaine étudié, ainsi que des méthodes heuristiques de résolution et des stratégies métacognitives; (5) favoriser une explicitation et une réflexion sur les activités d'apprentissage et sur les stratégies de résolution de problèmes. Ces principes sont au cœur de la présente étude. En effet, les tâches complexes en mathématiques rencontrent le troisième principe puisqu'il s'agit de problèmes ouverts, réalistes qui nécessitent la mobilisation de ressources dans une variété de contextes. Pour développer un enseignement/ apprentissage favorisant la résolution de tâches complexes, la théorie de De Corte et Verschaffel (2008) laisse penser qu'il serait porteur de parier simultanément sur le concept de co-régulation (régulations externes interactives et autorégulation: cf. principes 1 et 2) ainsi que sur une co-construction d'une démarche experte et réflexive de résolution de problèmes (cf. principes 4 et 5).

Allal (2007) définit la régulation comme l'ensemble des «mécanismes qui assurent le guidage, le contrôle, l'ajustement des activités cognitives, affectives et sociales, favorisant ainsi la transformation des compétences de l'apprenant» (p. 9). La régulation désigne à la fois les apports extérieurs qui facilitent l'apprentissage (régulations externes ou hétérorégulation) et le travail interne, métacognitif, de l'apprenant (régulation interne ou autorégulation). Les interventions externes ne sont que des «sources de régulations potentielles qui ont une fonction de médiation à l'autorégulation» (Mottier Lopez, 2012, p. 31). Autrement dit, pour qu'il y ait apprentissage, on ne peut parier que sur l'autorégulation qui, elle-même, est stimulée par une action éducative (Perrenoud, 1998a). Pour montrer à quel point les deux versants sont liés, Allal (2007) propose le concept de «co-régulation, mettant en relation des processus d'autorégulation avec des formes de guidages par des facteurs contextuels» (p. 11).

Parmi les démarches de régulation externe couramment identifiées, les régulations interactives semblent être les plus intéressantes dans le cadre d'une séance d'enseignement/apprentissage portant sur des tâches complexes. Intervenant au cœur même de l'activité, elles sont considérées comme des «régula- 
tions puissantes» (Mottier Lopez, 2012, p. 27). Allal (2007) distingue trois modes de régulation interactive: les interactions engageant le maitre et l'élève $(\mathrm{M}-\mathrm{E})$, les élèves entre eux (E-E), l'élève et les outils mis à sa disposition (E-O). Les régulations interactives $\mathrm{M}-\mathrm{E}$ sont très fréquentes en classe. L'enseignant ne peut pas «faire à la place» de l'élève, mais il juge indispensable de le stimuler, de le réorienter quand il constate une difficulté ou une erreur. Selon Perrenoud (1998a), cette démarche est une prise de risque car l'enseignant interfere dans «les processus de pensée et de communication en cours» (p. 114). Il est donc essentiel qu'il interprète correctement les démarches de l'élève pour lui fournir des indices appropriés à son raisonnement. Les régulations interactives E-E font, quant à elles, référence aux moments de travail entre pairs. De nombreuses recherches en ont souligné les gains cognitifs, sociaux et psychologiques, et ce particulièrement face à des tâches ouvertes et complexes susceptibles de favoriser une réelle coopération entre élèves (Buchs, Lerhaus \& Crahay, 2012). Enfin, les régulations interactives $\mathrm{E}-\mathrm{O}$ confrontant l'élève à un outil (référentiel, grille de co-évaluation, outil technologique...) peuvent s'avérer pertinentes pour soutenir les deux autres modes de régulation interactive. Le matériel fait office de support: les élèves peuvent, par exemple, s'en servir pour appuyer leurs propos ou justifier leur position.

En classe, l'enseignant facilite l'autorégulation nécessaire à l'apprentissage en construisant des dispositifs didactiques favorables au développement de démarches de régulations interactives. Cependant, compte tenu des difficultés que représente le déploiement de cette autorégulation, il doit également mener une "guidance appropriée», c'est-à-dire un enseignement plus explicite de stratégies cognitives (ou de méthodes heuristiques) visant à favoriser le développement de stratégies métacognitives propres à la résolution de tâches complexes. Plusieurs études (Montague, 2007; Özoy \& Ataman, 2009; Perels, Gürtler \& Schmitz, 2005) ont montré l'efficacité d'approches centrées sur l'enseignement de stratégies cognitives et métacognitives et/ou favorisant l'apprentissage autorégulé (self-regulation strategies) face à la résolution de problèmes mathématiques. Centrée sur la comparaison de plusieurs dispositifs didactiques, l'étude de Perels et al. (2005) montre la plus-value d'une approche combinant l'enseignement de stratégies d'autorégulation et de stratégies de résolution de problèmes, tout en pointant également l'efficacité d'approches isolant l'une ou l'autre de ces dimensions. Dans la même lignée, les travaux de Verschaffel, De Corte, Lasure, Van Vaerenbergh, Bogaerts et Ratinckx, (1999; voir aussi Van Dooren et al., 2015; Verschaffel \& De Corte, 2008) ont montré l'efficacité d'une approche consistant à considérer la résolution de problèmes comme «un processus complexe de modélisation mathématique» constitué de six phases: (1) la construction d'un modèle de situation traduisant la compréhension du phénomène à investiguer, (2) la construction d'un modèle mathématique, (3) l'exécution des opérations mathématiques, (4) l'interprétation des résultats, (5) l'évaluation de la solution avancée en référence au modèle de situation et (6) la 
communication de la solution interprétée. Une démarche experte et réflexive de résolution de problèmes consisterait alors à envisager ces six phases, non pas selon un ordre linéaire et immuable, mais en régulant sa démarche et en procédant aux allers-retours nécessaires pour mener à bien le processus. L'approche d'enseignement/apprentissage développée par cette équipe de recherche s'appuie sur des tâches complexes et authentiques et propose notamment un enseignement explicite d'heuristiques propres aux différentes phases du processus de résolution de problèmes (ex: faire un dessin ou un schéma, faire appel à ses connaissances de la vie réelle, simplifier les nombres, évaluer la pertinence de sa solution...) tout en insistant sur l'importance de la dimension réflexive (métacognitive) visant à favoriser l'autorégulation.

\section{Objectifs et questions de recherche}

Le premier objectif de la recherche était de développer, en collaboration avec une enseignante, un dispositif d'enseignement/apprentissage stimulant (De Corte \& Verschaffel, 2008), propre à permettre aux élèves de développer des compétences face à la résolution de tâches complexes en mathématiques. En accord avec la revue de la littérature développée au point précédent, ce dispositif devait favoriser les moments de régulations interactives (Allal, 2007) et le développement d'une démarche experte et réflexive de résolution de problèmes (Van Dooeren et al., 2015; Verschaffel \& De Corte, 2008). Ce dispositif a été conçu et mis en place dans une classe d'élèves de $\sigma^{\mathrm{e}}$ année primaire durant trois séances dévolues chacune à l'exploitation d'une tâche complexe de mathématiques. Il s'agissait ensuite d'évaluer l'efficacité de l'approche développée.

L'analyse sera guidée par trois questions de recherche, les deux premières portent sur l'implantation du dispositif, la troisième sur son efficacité: (1) le dispositif développé offre-t-il aux élèves l'occasion de s'engager dans une démarche experte et réflexive de résolution de problèmes? (2) permet-il de favoriser les moments de régulations interactives, sources potentielles d'autorégulation (co-régulation)? (3) les élèves qui ont bénéficié de ce dispositif progressent-ils au niveau de leurs compétences en résolution de problèmes?

\section{Dispositif de recherche}

Deux classes de 25 élèves de $6^{\mathrm{e}}$ primaire en Belgique francophone ont participé à la présente recherche. Celle-ci s'appuie sur un dispositif quasi-expérimental classique avec classe expérimentale, classe contrôle, prétest, phase d'intervention et post-test.

La phase d'intervention invitait les deux enseignants à organiser trois séances d'enseignement/apprentissage autour de trois tâches complexes, à raison d'une 
tâche par semaine. Ces tâches étaient issues des outils d'évaluation proposés sur le site du Ministère de l'enseignement comme exemples de tâches complexes en mathématiques et mettaient en jeu des ressources élémentaires diverses. Dans la classe contrôle, le professeur ne recevait aucune directive méthodologique: il lui était demandé d'utiliser les trois tâches complexes retenues, mais il avait la liberté d'organiser les leçons selon ses pratiques habituelles en résolution de problèmes. Dans la classe expérimentale, en revanche, l'enseignante appliquait le dispositif d'enseignement/apprentissage construit en collaboration avec la chercheuse en suivant une trame précisant les différentes étapes de la séance et les stratégies à mettre en œuvre pour favoriser le développement d'une démarche experte de résolution de problèmes et promouvoir différents moments de régulations interactives (voir l'analyse de l'implantation du dispositif au point 5.1).

Les séances de la phase d'intervention ont été filmées et retranscrites intégralement de façon à comparer les démarches de régulation interactive engagées ainsi que la façon dont la démarche experte de résolution de problèmes se (co-) construisait dans les classes.

Pour évaluer l'efficacité de l'approche, les prétest et post-test ${ }^{1}$ comprenaient une même tâche complexe (la phase 1 de l'outil d'évaluation de compétences intitulé "À l'affiche» ${ }^{2}$ ), ainsi que six problèmes ${ }^{3}$ tirés de l'épreuve externe organisée en fin de 6e année primaire pour l'obtention du Certificat d'Étude de Base $(\mathrm{CEB})^{4}$. La procédure de passation était identique dans les deux classes. Les élèves disposaient de leur matériel habituel, d'une calculatrice et d'une feuille de brouillon. Ils devaient résoudre la tâche complexe et les six problèmes en soixante minutes de travail individuel. La seule consigne donnée était de veiller à retranscrire toutes leurs démarches. Pour documenter davantage les progrès cognitifs, nous avons conduit un entretien semi-dirigé avec cinq élèves de chaque classe à la suite de chaque séance d'intervention. Il s'agissait de deux élèves «faibles», de deux élèves «moyens» et d'un élève "fort», choisis sur la base de leurs résultats au prétest.

\section{Résultats principaux}

\section{Analyse de l'implantation du dispositif expérimental}

Pour rendre compte des particularités du dispositif implanté dans la classe expérimentale, nous le présenterons en comparant les pratiques d'enseignement observées dans les deux classes ayant participé à l'étude. Comme le synthétise le tableau 1, les séances de la phase d'intervention se déroulent en trois étapes clés (le lancement de la tâche, la résolution, la mise en commun), mais la méthodologie et la dynamique engagées varient fortement d'une classe à l'autre. 
Tableau 1: Comparaison des méthodologies et dynamiques observées lors des séances de la phase d'intervention

\begin{tabular}{|c|c|c|}
\hline & Classe expérimentale & Classe contrôle \\
\hline $\begin{array}{l}\text { Étape 1: } \\
\text { Lancement } \\
\text { de la tâche }\end{array}$ & $\begin{array}{l}\text { Annonce des enjeux d'apprentissage; } \\
\text { Lecture collective de la tâche; } \\
\text { Planification individuelle à l'aide d'une } \\
\text { représentation de la tâche (dessin, } \\
\text { schéma...); } \\
\text { Mise en commun des représentations. }\end{array}$ & $\begin{array}{l}\text { Rappel des règles de fonctionnement; } \\
\text { Découverte individuelle de la tâche. }\end{array}$ \\
\hline $\begin{array}{l}\text { Étape 2: } \\
\text { Résolution } \\
\text { de la tâche }\end{array}$ & $\begin{array}{l}\text { Travail individuel avec/sans interactions } \\
\text { personnalisées avec le maitre (M-E); } \\
\text { Travail en sous-groupes hétérogènes } \\
\text { (E-E); } \\
\text { Indices distribués par le maitre aux élèves } \\
\text { en difficulté (E-O). }\end{array}$ & $\begin{array}{l}\text { Travail individuel; } \\
\text { Interventions collectives du maitre (M-E); } \\
\text { Indices affichés au TN par le maitre } \\
\text { (E-O). }\end{array}$ \\
\hline \multirow{4}{*}{$\begin{array}{l}\text { Étape 3: } \\
\text { Mise en } \\
\text { commun }\end{array}$} & Correction de la tâche & Correction de la tâche \\
\hline & $\begin{array}{l}\text { Guidage ouvert (priorité aux sollicitations } \\
\text { et aux réponses de développement); } \\
\text { Présentation et acceptation de toutes les } \\
\text { démarches possibles de résolution; } \\
\text { Autorité mathématique partagée entre le } \\
\text { maitre et les élèves (chacun doit justifier sa } \\
\text { démarche pour convaincre les autres). }\end{array}$ & $\begin{array}{l}\text { Guidage fermé (priorité aux explications } \\
\text { et aux réponses de restitution); } \\
\text { Présentation de la démarche de résolution } \\
\text { anticipée par le maitre; } \\
\text { Autorité mathématique réservée au maitre } \\
\text { (l'enseignant valide les démarches et les } \\
\text { solutions). }\end{array}$ \\
\hline & Construction de la démarche de résolution & Construction de la démarche de résolution \\
\hline & $\begin{array}{l}\text { Tout au long de la correction, l'enseignant } \\
\text { pointe les éléments clés de la démarche } \\
\text { de résolution et met l'accent sur quelques } \\
\text { heuristiques essentielles (faire un schéma, } \\
\text { faire appel aux connaissances de la vie } \\
\text { réelle, planifier les étapes avant de réaliser } \\
\text { les calculs, vérifier la plausibilité de la } \\
\text { solution...); } \\
\text { Grille de co-évaluation (critères basés } \\
\text { sur les étapes d'une démarche experte de } \\
\text { résolution de tâches complexes). }\end{array}$ & $\begin{array}{l}\text { Rappel, effectué par l'enseignant au terme } \\
\text { de la correction, de la démarche efficace } \\
\text { pour résoudre des problèmes. }\end{array}$ \\
\hline
\end{tabular}

En accord avec les intentions décrites dans les points précédents, les deux classes se distinguent essentiellement sur deux ensembles de variables: celles propres au développement d'une démarche experte de résolution de problèmes et celles visant à promouvoir différentes formes de régulations interactives.

Concernant le développement d'une démarche experte et réflexive de résolution, la classe expérimentale se différencie de la classe contrôle par trois éléments majeurs:

- une analyse préalable de la tâche lors de la phase introductive via une démarche de planification à l'aide d'une représentation schématique ou dessinée (heuristique propre à la démarche experte de résolution);

- une focalisation sur les étapes clés d'une démarche experte de résolution et sur quelques heuristiques propres à ces étapes lors de la phase de mise en commun;

- l'utilisation d'une grille de co-évaluation proposée à la fin de chaque séquence de cours en vue d'aider les élèves à se situer dans leur acquisition de la démarche experte de résolution. 
La grille de co-évaluation explicitait les différentes étapes à mettre en œuvre pour résoudre un problème (de la construction d'un modèle de situation jusqu'à la communication d'un résultat interprété et à l'évaluation de sa pertinence, Verschaffel \& De Corte, 2008) et encourageait une réflexion métacognitive (Mevarech \& Kramarski, 2014). Après avoir rempli la grille d'auto-évaluation, les élèves obtenaient un feedback de l'enseignant qui remplissait également la grille et la complétait par des conseils relatifs aux aspects de la démarche jugés "problématiques». Ces feedbacks s'appuyaient sur des stratégies cognitives (heuristiques) visant à soutenir les différentes étapes du processus de résolution et à encourager l'élève (par ex. "N'hésite pas à lire plusieurs fois l'énoncé et à faire un dessin ou un schéma pour t'aider à voir comment procéder!»; "Il faut vraiment que tu penses à vérifier que tes réponses soient plausibles... C'est important."; "Bravo! C'est toi qui as pensé à rappeler au groupe qu'il fallait rédiger une phrase pour indiquer la réponse finale. Continue!»). Avant d'aborder une nouvelle tâche complexe, l'élève devait, sur la base des feedbacks reçus, choisir un (ou deux) élément(s) qu'il s'engageait à améliorer lors de la séance suivante.

Pour mieux cerner les différentes formes de régulations interactives à l'œuvre durant la leçon, l'analyse qui suit se centre essentiellement sur la deuxième étape des séquences d'enseignement (Étape 2 - «Résolution de la tâche»).

Dans la classe expérimentale, deux modalités de travail sont proposées: un temps de travail individuel où l'enseignante peut s'entretenir avec les élèves en difficulté (M-E), puis un temps de travail en groupes hétérogènes (E-E) lorsque les élèves ont amorcé une démarche de résolution. Lors de cette phase, l'enseignante propose, si nécessaire, les indices anticipés (E-O) avant la séance sur la base d'une analyse apriori de la tâche. Trois types d'indices étaient disponibles: des questions pour relancer la réflexion, des supports visuels soutenant la construction d'une représentation de la tâche et des exemples de raisonnements erronés visant à favoriser la mise en œuvre d'un processus de vérification. Dans la classe contrôle, l'enseignant privilégie le travail individuel et intervient ponctuellement pour livrer des informations à la classe (M-E). Il a également prévu des indices (E-O) pour faciliter la résolution des tâches. Cependant, l'objectif poursuivi par ceux-ci, leur utilisation et leurs caractéristiques étaient distincts de ceux de la classe expérimentale, comme l'illustre le tableau 2.

Tableau 2: Comparaison de l'utilisation, des caractéristiques et des objectifs des indices proposés dans les classes expérimentale et contrôle

\begin{tabular}{|l|l|l|}
\cline { 2 - 3 } \multicolumn{1}{c|}{} & Classe expérimentale & Classe contrôle \\
\hline Utilisation & $\begin{array}{l}\text { Indices proposés aux groupes d'élèves } \\
\text { lorsqu'ils rencontrent une difficulté } \\
\text { spécifique. }\end{array}$ & $\begin{array}{l}\text { Indices proposés à l'ensemble de la classe } \\
\text { de manière ponctuelle. }\end{array}$ \\
\hline $\begin{array}{l}\text { Caractéris- } \\
\text { tiques }\end{array}$ & $\begin{array}{l}\text { Indices principalement ciblés sur le } \\
\text { processus de résolution (questions pour } \\
\text { relancer la réflexion, supports visuels, } \\
\text { raisonnements erronés). }\end{array}$ & $\begin{array}{l}\text { Indices principalement ciblés sur le } \\
\text { produit (éléments clés de la résolution, } \\
\text { aides à la présentation de la réponse). }\end{array}$ \\
\hline
\end{tabular}




\begin{tabular}{|l|l|l|}
\hline Objectifs & $\begin{array}{l}\text { Aider les élèves en difficulté à réguler leur } \\
\text { démarche de résolution; } \\
\text { Soutenir les interactions (E-E). }\end{array}$ & $\begin{array}{l}\text { Aider les élèves en difficulté à réguler leur } \\
\text { démarche de résolution. }\end{array}$ \\
\hline
\end{tabular}

Dans les deux classes, les enseignants montrent leur volonté d'aider les élèves tout en les laissant chercher, construire seuls une démarche de résolution. Cependant, plusieurs différences dans leur fonctionnement sont à souligner. La figure 1 contraste les dynamiques de travail instaurées lors de la dernière séance d'intervention.

\section{Figure 1: Transcription des échanges pendant la résolution individuelle de la $3 e$ tâche complexe}

Mise en contexte: la tâche invite l'élève à tracer un circuit dans la ville de Namur permettant de relier cinq curiosités, mais aussi à calculer le temps nécessaire pour le parcourir en prévoyant un arrêt de 10 minutes devant chaque curiosité.

\section{$\rightarrow$ Classe expérimentale}

E1: Madame, on ne sait pas vraiment combien de temps ça prend pour faire le trajet...

M: Ça, je ne peux pas te dire...

E1: Oui, je sais, mais je veux dire, par exemple, on ne sait pas combien de temps ça va prendre du premier arrêt au deuxième arrêt... On ne sait pas vraiment le dire puisqu'ils ne le disent pas dans les feuilles.

M: Ah oui. Peut-être que tu pourrais réfléchir et trouver toi-même cette information... Courage!

M. s'en va et part observer d'autres Es. (...)

E2: Madame, on ne pourrait pas travailler en groupes?

M: On doit d'abord réfléchir seul avant d'aller en groupes.

E2 regarde sa feuille, il semble bloqué, puis se remet à chercher. 2 minutes plus tard...

E2: Madame, ils ne nous disent pas le temps que ça prend entre chaque curiosité...

M: Non, on ne le dit pas.

E2: Alors, c'est impossible. On ne sait pas le faire.

M: $S i$, on peut... Réfléchis par toi-même à quelque chose qui pourrait t'aider... De quelle information as-tu besoin?

E2: Euh... Au fait, ils marchent à pied?

M: Circuit pédestre. Ça veut dire quoi "pédestre»?

E3 entend la question et intervient.

E3: Ça ne veut pas dire "pied» en grec ou...?

M: Oui, il y a surement une racine grecque ou latine qui signifie pied. E2: Ah oui, donc c'est tout à pied, ils marchent à pied...!

\section{$\rightarrow$ Classe contrôle}

M: Petit indice supplémentaire: nous marchons à $4 \mathrm{~km} / \mathrm{h}$, plus ou moins... Normalement, avec tout ce que je vous ai donné, je ne dois plus rien dire... Le tableau des mesures, vous le connaissez, j'espère? Vous savez que $1 \mathrm{~km}$, c'est... combien de mètres?

Es: 1000 !

M: Oui, donc à partir du moment où vous connaissez votre tableau et que vous avez ces indices-là, je ne vous suis plus d'une grande utilité.
M. suscite la réflexion chez E1 en sous-entendant que l'information doit être trouvée par réflexion et non en cherchant dans les documents.

M. encourage (contrôle de la frustration).

E2 voudrait solliciter le groupe car il est conscient d'une difficulté liée à la tâche: trouver la vitesse de marche.

M. soutient la réflexion d'E2 (régulation des processus cognitifs).

E2 semble avoir compris une information importante pour sa démarche de résolution.

Intervention ponctuelle destinée à l'ensemble des Es.

Volonté de laisser les Es se "débrouiller". 
Lors de la phase de travail individuel, les régulations maitre-élève (M-E) observées dans les deux classes sont de nature différente. Dans la classe expérimentale, elles sont destinées à solliciter la réflexion des élèves concernés par une difficulté. En effet, l'enseignante n'hésite pas à les faire verbaliser (par ex. "que fais-tu?"; "comment comptes-tu t'y prendre?») et à leur apporter un soutien dans les démarches engagées. En revanche, dans la classe contrôle, les quelques interactions entre le maitre et les élèves sont réalisées collectivement. Les remarques sont formulées à voix haute. Ainsi, il suscite moins la réflexion de l'élève face à ses propres démarches, mais privilégie les «explications» collectives.

Dans la classe expérimentale, le temps de travail en groupes hétérogènes favorise les régulations entre élèves $(\mathrm{E}-\mathrm{E})$. Les échanges donnent lieu à des conflits socio-cognitifs les conduisant à clarifier leur position (en créant des liens avec la vie quotidienne, en se fondant sur des éléments du document...) et à comprendre celle des autres. Les arguments de chacun sont analysés, complétés, voire totalement modifiés. Ensemble, les élèves parviennent à une solution commune et généralement pertinente car elle a fait l'objet de nombreuses réflexions. Le fait d'être en groupe aide également les élèves à gérer la complexité des tâches, échappant sans doute à une éventuelle surcharge cognitive: ils se corrigent, se rappellent les étapes du problème, les démarches efficaces à mettre en œuvre...

Les interactions entre élèves sont soutenues par de nombreuses régulations maitre-élève(s). L'enseignante de la classe expérimentale tente régulièrement de réguler l'activité des élèves (en les incitant à respecter les normes de fonctionnement de l'apprentissage par pairs), mais aussi leurs processus cognitifs (en les invitant à expliciter leurs raisonnements, à confronter les avis...). De manière générale, des discussions autour des éléments importants d'une démarche experte de résolution de tâches complexes (exemple: nécessité de lire toutes les informations disponibles) sont aussi engagées. Connaissant les objectifs que les élèves se fixent grâce à la grille de co-évaluation, l'enseignante attire leur attention sur ceux-ci et veille à ce qu'ils les respectent.

Enfin, le recours à une régulation élève-outil (E-O) est présent dans les deux classes. Cette dernière est largement privilégiée dans la classe contrôle où l'enseignant n'hésite pas à indiquer aux élèves en difficulté la présence d'indices affichés au tableau sans engager d'échanges personnalisés. Dans la classe expérimentale, les indices sont principalement proposés aux élèves lors des échanges en groupes hétérogènes. Généralement, ils s'avèrent utiles pour aider les pairs à résoudre les conflits socio-cognitifs ou pour dépasser les difficultés liées à la tâche. Ils peuvent servir de support pour appuyer leurs explications, pour les encourager à s'engager dans des stratégies de contrôle ou encore pour les inviter à mettre en œuvre une stratégie (faire un dessin, utiliser ses connaissances antérieures, etc.). La figure 2 illustre l'efficacité d'un indice visuel donné lors de la deuxième séance. 
Figure 2: L'indice engage les élèves dans une stratégie de contrôle et leur inspire une stratégie

Mise en contexte: la tâche invite l'élève à calculer le coût total de l'aménagement d'un jardin. Il doit, entre autres, prévoir de planter 20 narcisses par mètre carré dans un parterre de forme triangulaire.

$\rightarrow$ TC2: côté cour, côté jardin

Les Es ont calculé le prix de 20 narcisses, mais s'interrogent... E4: On demande 20 narcisses par mètre carré donc il faut en acheter...

E3: Oui, par mètre carré, mais...

Les enfants semblent bloqués.

M: Regardez ceci!

E4: Ah! Regardez, il y a plus qu'1 $m^{2}$ là-dedans!

E3: Oui, $1 m^{2}=20$ narcisses, un autre $m^{2}=20$ narcisses, tout le temps comme ça!

M: C'est quoi $1 m^{2}$ ?

E4: $1 \mathrm{~m}$ sur $1 \mathrm{~m}$ !

E3: Et il y a 20 narcisses dedans!

M: E1, tu es d'accord avec ça?

E1: Oui, en fait, on ne doit pas acheter 20 narcisses, mais le nombre de narcisses qu'il faut par mètre carré...

E5: Ah! Il y a 20 narcisses "par mètre carré»!

Les enfants cherchent à savoir combien de mètres carrés il y a dans le parterre triangulaire.

E4: $3 m^{2}$ !

E3: Non, il y en a plus regarde! $\mathbf{E} 3$ commence à dessiner des carrés dans le parterre de forme triangulaire.

E4: Attends, il y a ... $6 m^{2}$ !

E3: Moi, j'aurais dit qu'il y en a 5!

E2: T'es sûr qu'il y en a 5 ou c'est juste comme ça? Comment tu fais? E3: Il faut dessiner les mètres carrés dans le triangle, regarde. Là il y en a 2, puis là, il n'est pas entier donc pour l'avoir, on devra rajouter ce bout-là.

M: Comment pourrait-on faire pour être plus précis?

E4: Moi, j'ai une idée, super facile! On va faire "fois 2" pour faire un carré. On calcule les mètres carrés, puis on divisera par 2!

En suivant la suggestion d'E4, les enfants ont représenté facilement les petits carrés d' $1 \mathrm{~m}^{2}$ dans le carré. Ils en ont trouvé 9 et ont divisé par deux (réponse exacte).

\section{Analyse de l'efficacité du dispositif développé dans la classe expérimentale}

En vue d'apporter quelques indicateurs visant à appréhender l'efficacité du dispositif d'enseignement/apprentissage présenté aux pages qui précèdent, cette partie s'intéresse aux progrès réalisés dans les deux classes entre le pré et le post-test. Elle présente aussi quelques résultats issus des entretiens en vue de documenter les progrès cognitifs réalisés par quelques élèves de chaque classe tout au long des trois séances.

\section{Comparaisons prétest-post-test}

La tâche complexe "À l'affiche!» demande d'établir l'horaire d'une sortie au cinéma pour une classe d'élèves et d'en calculer le prix le plus avantageux par 
élève. La tâche complexe comporte donc deux sous-tâches: (1) réaliser l'horaire et (2) calculer le coût total de la sortie au cinéma. Chaque sous-tâche nécessite la mise en œuvre de plusieurs étapes de résolution. Chaque élève est noté sur un total de 16 points pour la rédaction de l'horaire 5 et sur un total de 10 points 6 pour le calcul du coût total. Le tableau 3 présente les résultats globaux obtenus par les deux classes aux deux sous-tâches de la tâche complexe lors du prétest et du post-test.

Tableau 3: Comparaison des résultats obtenus au prétest et au post-test face à la tâche complexe

\begin{tabular}{|c|c|c|c|c|c|c|c|c|c|}
\hline \multicolumn{5}{|c|}{ Rédaction de l'horaire } & \multicolumn{5}{|c|}{ Calcul du coût total } \\
\hline \multirow[t]{2}{*}{ Score } & \multicolumn{2}{|c|}{$\begin{array}{c}\text { Classe } \\
\text { expérimentale }\end{array}$} & \multicolumn{2}{|c|}{$\begin{array}{l}\text { Classe } \\
\text { contrôle }\end{array}$} & \multirow[t]{2}{*}{ Score } & \multicolumn{2}{|c|}{$\begin{array}{c}\text { Classe } \\
\text { expérimentale }\end{array}$} & \multicolumn{2}{|c|}{$\begin{array}{c}\text { Classe } \\
\text { contrôle }\end{array}$} \\
\hline & Prétest & Post-test & Prétest & Post-test & & Prétest & Post-test & Prétest & Post-test \\
\hline 16 & $\begin{array}{l}9.1 \% \\
(2 \mathrm{Es})\end{array}$ & $\begin{array}{c}22.7 \% \\
(5 \mathrm{Es})\end{array}$ & $\begin{array}{l}0 \% \\
(0 \mathrm{E})\end{array}$ & $\begin{array}{l}0 \% \\
(0 \mathrm{E})\end{array}$ & & & & & \\
\hline$[15-12]$ & $\begin{array}{l}13.6 \% \\
(3 \mathrm{Es})\end{array}$ & $\begin{array}{l}31.8 \% \\
\text { (7 Es) }\end{array}$ & $\begin{array}{l}12.5 \% \\
(3 \mathrm{Es})\end{array}$ & $\begin{array}{l}20.8 \% \\
(5 \mathrm{Es})\end{array}$ & 10 & $\begin{array}{c}22.7 \% \\
(5 \mathrm{Es})\end{array}$ & $\begin{array}{l}36.3 \% \\
(8 \mathrm{Es})\end{array}$ & $\begin{array}{l}4.2 \% \\
(1 \mathrm{E})\end{array}$ & $\begin{array}{l}8.3 \% \\
(2 \mathrm{Es})\end{array}$ \\
\hline$[11-8]$ & $\begin{array}{c}31.8 \% \\
(7 \mathrm{Es})\end{array}$ & $\begin{array}{c}27.3 \% \\
(6 \mathrm{Es})\end{array}$ & $\begin{array}{l}16.7 \% \\
(4 \mathrm{Es})\end{array}$ & $\begin{array}{l}4.2 \% \\
(1 \mathrm{E})\end{array}$ & {$[9-7]$} & $\begin{array}{c}27.3 \% \\
(6 \mathrm{Es})\end{array}$ & $\begin{array}{l}27.3 \% \\
(6 \mathrm{Es})\end{array}$ & $\begin{array}{l}25.0 \% \\
(6 \mathrm{Es})\end{array}$ & $\begin{array}{l}37.5 \% \\
(9 \mathrm{Es})\end{array}$ \\
\hline$[7-4]$ & $\begin{array}{l}13.6 \% \\
(3 \mathrm{Es})\end{array}$ & $\begin{array}{l}9.1 \% \\
(2 \mathrm{Es})\end{array}$ & $\begin{array}{l}41.6 \% \\
(10 \mathrm{Es})\end{array}$ & $\begin{array}{l}33.3 \% \\
(8 \mathrm{Es})\end{array}$ & {$[6-4]$} & $\begin{array}{l}18.2 \% \\
(4 \mathrm{Es})\end{array}$ & $\begin{array}{l}27.3 \% \\
(6 \mathrm{Es})\end{array}$ & $\begin{array}{l}41.6 \% \\
(10 \mathrm{Es})\end{array}$ & $\begin{array}{l}25.0 \% \\
(6 \mathrm{Es})\end{array}$ \\
\hline$\leq 3$ & $\begin{array}{c}31.8 \% \\
(7 \mathrm{Es})\end{array}$ & $\begin{array}{l}9.1 \% \\
(2 \mathrm{Es})\end{array}$ & $\begin{array}{l}29.2 \% \\
(7 \mathrm{Es})\end{array}$ & $\begin{array}{l}41.6 \% \\
(10 \mathrm{Es})\end{array}$ & $\leq 3$ & $\begin{array}{l}31.8 \% \\
(7 \mathrm{Es})\end{array}$ & $\begin{array}{l}9.1 \% \\
(2 \mathrm{Es})\end{array}$ & $\begin{array}{l}29.2 \% \\
(7 \mathrm{Es})\end{array}$ & $\begin{array}{l}29.2 \% \\
(7 \mathrm{Es})\end{array}$ \\
\hline $\begin{array}{l}\text { Score } \\
\text { moyen }\end{array}$ & $\begin{array}{c}43.7 \% \\
(7 \text { sur } \\
16)\end{array}$ & $\begin{array}{c}67.5 \% \\
(10.8 \\
\text { sur } 16)\end{array}$ & $\begin{array}{c}33.1 \% \\
(5.3 \mathrm{sur} \\
16)\end{array}$ & $\begin{array}{c}31.9 \% \\
(5.1 \mathrm{sur} \\
16)\end{array}$ & $\begin{array}{l}\text { Score } \\
\text { moyen }\end{array}$ & $\begin{array}{c}54.0 \% \\
(5.4 \text { sur } \\
10)\end{array}$ & $\begin{array}{c}73.0 \% \\
(7.3 \\
\text { sur } 10)\end{array}$ & $\begin{array}{c}50.0 \% \\
(5 \text { sur } \\
10)\end{array}$ & $\begin{array}{c}54.0 \% \\
(5.4 \text { sur } \\
10)\end{array}$ \\
\hline $\begin{array}{l}\text { Gain } \\
\text { relatif }\end{array}$ & & & & & $\begin{array}{l}\text { Gain } \\
\text { relatif }\end{array}$ & & & & $\%$ \\
\hline
\end{tabular}

Les résultats montrent des progrès nettement supérieurs dans la classe expérimentale. Pour l'horaire, le gain relatif est supérieur à $40 \%$, alors qu'il est légèrement négatif dans la classe contrôle. Pour le calcul du coût, le gain relatif est plus de cinq fois supérieur à celui de la classe contrôle. Alors que, dans les deux classes, $30 \%$ des élèves présentaient un score très faible (égal ou inférieur à 3) au prétest, seuls $10 \%$ des élèves de la classe expérimentale restent faibles à l'issue du post-test, contre un peu plus de $40 \%$ des élèves pour la sous-tâche «horaire» et $30 \%$ pour celle du calcul du coût dans la classe contrôle. 
Le tableau 4 présente les résultats obtenus aux problèmes du CEB.

Tableau 4: Comparaison des proportions d'élèves ayant réussi les différents problèmes $d u C E B$ au prétest et au post-test

\begin{tabular}{|c|c|c|c|c|c|c|}
\cline { 2 - 7 } \multicolumn{1}{c|}{} & \multicolumn{3}{c|}{ Classe expérimentale $(\mathrm{N}=\mathbf{2 2})$} & \multicolumn{3}{c|}{ Classe contrôle $(\mathrm{N}=\mathbf{2 5})$} \\
\cline { 2 - 7 } \multicolumn{1}{c|}{} & Prétest & Post-test & Gain relatif & Prétest & Post-test & Gain relatif \\
\hline P1 & $46 \%$ & $91 \%$ & $\mathbf{8 3 \%}$ & $68 \%$ & $48 \%$ & $\mathbf{- 6 3 \%}$ \\
\hline P2 & $77 \%$ & $91 \%$ & $\mathbf{6 0} \%$ & $44 \%$ & $60 \%$ & $\mathbf{2 9 \%}$ \\
\hline P3a & $36 \%$ & $55 \%$ & $\mathbf{2 9} \%$ & $20 \%$ & $20 \%$ & $\mathbf{0 \%}$ \\
\hline P3b & $36 \%$ & $64 \%$ & $\mathbf{4 3 \%}$ & $32 \%$ & $12 \%$ & $\mathbf{- 2 9 \%}$ \\
\hline P4 & $5 \%$ & $41 \%$ & $\mathbf{3 8} \%$ & $8 \%$ & $12 \%$ & $\mathbf{4 \%}$ \\
\hline P5 & $14 \%$ & $41 \%$ & $\mathbf{3 2} \%$ & $20 \%$ & $12 \%$ & $\mathbf{- 1 0 \%}$ \\
\hline P6 & $27 \%$ & $55 \%$ & $\mathbf{3 8} \%$ & $16 \%$ & $16 \%$ & $\mathbf{0 \%}$ \\
\hline
\end{tabular}

Là encore, la classe expérimentale se démarque largement, témoignant sans doute d'un réinvestissement de la démarche apprise face aux tâches complexes vers des problèmes plus classiques (tous les gains sont nettement positifs). À l'inverse, les résultats sont préoccupants dans la classe contrôle, puisqu'on note un gain positif marqué pour un seul problème et des gains négatifs pour trois d'entre eux.

\section{Apport des entretiens semi-dirigés}

Les entretiens semi-dirigés apportent un éclairage supplémentaire sur les progrès réalisés par cinq élèves de chaque classe (deux élèves faibles, deux élèves moyens et un élève fort). À la fin de chaque séance, la chercheuse a demandé aux élèves sélectionnés de refaire (oralement) la tâche qu'ils venaient de résoudre en classe. Le tableau 5 présente les résultats de ces entretiens.

Tableau 5: Comparaison des parts relatives d'élèves des classes expérimentale et contrôle capables de résoudre la tâche complexe à l'issue de la séance d'intervention correspondante

\begin{tabular}{|l|rl|rl|}
\cline { 2 - 5 } \multicolumn{1}{c|}{} & \multicolumn{3}{|c|}{ Classe expérimentale } & \multicolumn{3}{c|}{ Classe contrôle } \\
\hline TC1: les champions du pentathlon & $100 \%$ & $(5 E s)$ & $0 \%$ & $(O E)$ \\
\hline TC2: côté cour, côté jardin & $100 \%$ & $(5 E s)$ & $25 \%$ & $\left(1 E^{1}\right)$ \\
\hline TC3: circuit pédestre & $100 \%$ & $(5 E s)$ & $60 \%$ & $\left(3 E_{s}^{2}\right)$ \\
\hline
\end{tabular}

1 Il s'agit de l'élève «fort».

2 Il s'agit de l'élève «fort» et des deux élèves «faibles».

Dans la classe expérimentale, les cinq enfants sont capables de réussir chacune des tâches complexes de la phase d'intervention quel que soit leur niveau initial. Les résultats sont moins encourageants dans la classe contrôle et ce, surtout pour les 
enfants plus «faibles». S'ils ont parfois retenu la réponse finale, ils sont incapables de mettre en œuvre correctement une démarche leur permettant d'y aboutir.

\section{Discussion et conclusion}

L'approche mise en ouvre dans cette recherche collaborative a permis le développement et l'implantation d'un dispositif d'enseignement/apprentissage qui, en confrontant les élèves à des tâches complexes de mathématiques telles que définies par les tenants de l'approche par compétences (Rey et al., 2006), leur a permis non seulement de s'engager dans une démarche experte et réflexive de résolution de problèmes (Verschaffel \& De Corte, 2008), mais aussi d'en cerner explicitement les éléments essentiels et partant, d'en acquérir sans doute une certaine maîtrise. Pensé de façon à maximiser les occasions de régulations interactives (Allal, 2007), le dispositif didactique développé semble avoir permis une certaine forme de co-régulation, élément susceptible d'expliquer les progrès substantiels observés entre le prétest et le post-test tant au niveau de la tâche complexe (outil d'évaluation) que des problèmes arithmétiques plus classiques (problèmes issus du CEB).

Selon Mottier Lopez (2012, p. 84), les enseignants manquent essentiellement d'informations sur leur «rôle de médiation» lorsqu'ils proposent des tâches mathématiques aux élèves. L'observation des pratiques de l'enseignant de la classe contrôle semble, en effet, corroborer ces propos ${ }^{7}$ : à de nombreuses reprises, il valorise l'autonomie en marquant sa volonté de laisser l'élève gérer lui-même sa démarche de résolution. Durant cette phase, il n'interagit pas personnellement avec les élèves en difficulté et ne propose aucun moment d'échanges entre élèves. L'élève est seul face à la tâche imposée et doit réguler ses apprentissages en utilisant, si nécessaire, les indices (E-O) affichés. Ces derniers permettent à l'enseignant d'aider l'élève sans avoir le sentiment d'influencer trop fortement sa démarche de résolution. Cependant, les résultats du groupe contrôle sont peu encourageants, ce qui souligne l'importance d'un rôle plus actif de l'enseignant. Les régressions constatées pourraient s'expliquer par une perte de motivation de certains élèves. Il est, en effet, possible que ceux qui, tout au long des séances, ne parviennent pas à résoudre une tâche complexe finissent par se décourager et fournissent moins d'efforts lors du post-test. Cette hypothèse interprétative prend sens dans la mesure où l'influence des variables motivationnelles (sentiment de compétence et peur de l'échec notamment) face à la résolution de problèmes a été largement démontrée dans la littérature (Marcoux, 2014), mais aucune prise d'information en ce sens ne nous permet toutefois de l'étayer davantage.

Dans les deux classes, la présence d'indices (différents par leurs caractéristiques et leur utilisation) permet d'approfondir la réflexion sur la modalité de régulation interactive soutenue par des outils externes (E-O). En effet, les indices de la classe contrôle sont centrés sur le produit (éléments clés de la résolution, 
aides à la présentation de la réponse) plutôt que sur le processus (questions pour relancer la réflexion, supports visuels, raisonnements erronés...). Dans la classe expérimentale, en revanche, les indices ont été conçus pour éviter cette dérive: ils sont axés sur la représentation de la situation et non sur le produit ou sur une démarche spécifique vers laquelle orienter les élèves. Il s'agissait d'éviter de "dénaturer» le problème et d'influencer de manière trop directe la démarche à mettre en œuvre; il convenait «d'agir aussi légèrement que possible et de préserver de tout guidage le versant action du processus de résolution de problèmes» (Julo, 1995, p. 151). Ainsi, les indices, non seulement soutiennent les interactions et participent à un progrès cognitif, mais ils contribuent aussi à la construction de la démarche experte de résolution en induisant des stratégies cognitives (via l'utilisation d'heuristiques propres à certaines phases de la démarche de résolution comme le recours au dessin ou aux connaissances antérieures par exemple) et métacognitives (comme les stratégies planification et de vérification notamment).

Par ailleurs, l'utilisation des indices varie d'une classe à l'autre. Dans le groupe contrôle, après avoir observé une démarche erronée ou une difficulté chez l'un ou l'autre élève, l'enseignant expose un indice identique à l'ensemble de la classe et ce, au même moment. Dans le groupe expérimental, les indices sont donnés aux élèves à un moment jugé opportun par l'enseignante. Les enfants prêtent donc attention à l'information qui leur est proposée et l'utilisent pour contrôler leur démarche, soutenir leur raisonnement, dépasser un conflit... Le message de l'enseignante a plus de chance d'aboutir, car il s'insère dans le processus en cours et tente de trouver une entrée dans le système cognitif de l'apprenant (Perrenoud, 1998b).

Les deux classes se distinguent également quant aux modalités d'acquisition d'une stratégie experte de résolution de problèmes (Verschaffel \& De Corte, 2008). L'étape de mise en commun indique que l'enseignant de la classe contrôle parie sur un enseignement explicite de la démarche tandis que, dans la classe expérimentale, cette pratique est complétée par d'autres modalités d'action. Dans l'étape de résolution, des discussions sur l'importance de certaines stratégies cognitives et métacognitives sont engagées. Lors de l'étape de mise en commun, les élèves confrontent leurs démarches, expliquent et justifient leur propre raisonnement, cherchent à comprendre ceux des autres. De plus, les observations menées montrent que l'utilisation de la grille de co-évaluation permet aux élèves d'apprendre les stratégies cognitives et métacognitives: en s'auto-évaluant, elle les conduit à réfléchir à leur fonctionnement cognitif et les élèves régulent leurs actions en fonction des objectifs fixés. Cet outil permet également à l'enseignant de mieux connaître les difficultés des élèves et de mieux cibler ses interventions. L'approche semble ainsi avoir permis de travailler conjointement le développement de stratégies métacognitives et d'une démarche efficace de résolution de problèmes, combinaison d'éléments qui semble s'avérer très efficace, comme l'avaient déjà montré Perels et al. (2005) notamment. 
L'approche par compétences est souvent critiquée pour les risques d'inéquité qu'elle pourrait engendrer (Beckers, 2012; Chenu et al., 2014). Les entretiens montrent que les cinq élèves cibles de la classe expérimentale sont capables de refaire seuls les tâches complexes résolues au cours des trois séances alors que les élèves faibles de la classe contrôle ne parviennent pas à démontrer leur compréhension des apprentissages réalisés lors des séances. Ces résultats ne sont sans doute pas sans lien avec la dynamique instaurée dans la classe contrôle où le retrait de l'enseignant (dans le but de laisser les élèves autonomes dans la résolution) implique de facto un manque de différenciation dans ses pratiques. Ses interventions s'adressent généralement au collectif et sont donc moins personnalisées que dans la classe expérimentale.

L'étude exploratoire présentée dans cet article avait l'ambition de tester un dispositif d'enseignement/apprentissage visant à aider des élèves de $\sigma^{\mathrm{e}}$ primaire à résoudre des tâches complexes de mathématiques. En développant une approche mixte, combinant analyse qualitative des interactions en classe et dispositif quasi-expérimental, elle tentait de s'intéresser, comme le préconise Allal (2011), tant aux processus qu'aux produits d'apprentissage. Le dispositif mis en ouvre dans la classe expérimentale présente une efficacité certaine, même s'il n'est pas possible d'identifier si les progrès constatés sont dus à l'ensemble du dispositif ou à des aspects spécifiques de celui-ci. En d'autres termes, l'étude ne permet pas de cerner précisément quels sont les réels facteurs déclencheurs des apprentissages observés. En ce sens, elle laisse partiellement ouverte la question du "processus d'apprentissage» qui a permis aux élèves de développer la (mystérieuse) capacité de «mobiliser et intégrer» au cœur de l'approche par compétences (Rey et al., 2006).

Les résultats obtenus ouvrent des perspectives prometteuses en montrant que la résolution de tâches complexes n'est pas hors de portée des élèves, y compris les plus faibles, à condition de fournir un étayage adéquat. Elle offre des pistes d'actions aux enseignants et les invite à renoncer à toute forme d'inactivité au nom d'une certaine «dévolution didactique» qui peut conduire à parier sur une "autorégulation spontanée des apprenants» (Mottier Lopez, 2012, p. 92). Alors que le matériel didactique était le même au départ (les trois mêmes tâches complexes), les résultats contrastés observés dans les deux classes mettent en exergue le rôle essentiel de l'enseignant pour faire évoluer le raisonnement mathématique des élèves (Cobb, Zhao \& Visnovska, 2008). En ce sens, le développement professionnel des enseignants est sans doute une des pistes essentielles à creuser dans les recherches futures pour que les apprentissages mathématiques soient accessibles à tous les élèves. 


\section{Notes}

1 Nous avons fait le choix de proposer des tâches identiques au prétest et post-test. Comme mentionné précédemment, les tâches complexes nécessitent plusieurs étapes de résolution et de mobiliser divers documents tels que des horaires, des programmes, des tarifs... Construire des tâches comparables aurait nécessité d'utiliser le même type de documents (voire les mêmes documents) et de proposer les mêmes questionnements nécessitant les mêmes étapes de résolution. Puisque nous avons veillé à ce que les enseignants des deux classes ne reviennent nullement sur l'épreuve du prétest (pas de discussion sur les tâches réalisées et, évidemment, pas de correction de celles-ci) et puisqu'un délai d'un mois s'est écoulé entre les prétest et post-test, proposer des tâches identiques semblait la solution la plus adéquate pour permettre une réelle comparabilité des résultats.

2 La phase 1 de la tâche complexe "À l'affiche» est disponible sur le site www.enseignement. be dans la rubrique «Outils d'évaluation» de la deuxième étape de l'enseignement fondamental.

3 Au sein du test, les problèmes 1, 2 et 3 étaient issues du CEB 2012 (respectivement les questions 1,7 et 11 du livret 4), le problème 4 du CEB 2011 (question 2 du livret 4) et les problèmes 5 et 6 du CEB 2008 (question 12 du livret 5, question 10 du livret 1). Les épreuves du CEB sont accessibles sur le site www.enseignement.be dans la rubrique «Évaluations externes certificatives» (Accueil > De A à Z > Évaluations > Évaluations externes certificatives $>$ CEB $>$ Épreuves).

4 Contrairement aux tâches complexes provenant des outils d'évaluations de compétences qui impliquent de gérer divers documents, d'organiser les différentes étapes nécessaires à la résolution et, pour ce faire, de mobiliser et d'intégrer une série de procédures mathématiques, les problèmes issus du CEB sont des problèmes arithmétiques «classiques» nettement moins ouverts et complexes. En d'autres termes, alors que les premières correspondent aux définitions ambitieuses des compétences défendues par certains auteurs (compétences de troisième degré ou réelles compétences selon Rey et al., 2006, dont les travaux ont largement influencé les concepteurs des outils), les secondes s'approchent davantage de ce que ces mêmes auteurs qualifient de "compétences de deuxième degré» et d'autres «d'application habillées» (De Ketele, 2010).

5 Un horaire complet comptant huit étapes, un point a été accordé par étape envisagée et un point a été accordé par étape correcte.

6 Un maximum de six points était accordé pour l'identification des données correctes. Trois points étaient accordés pour la simple identification à raison d'un point par donnée (nombre d'élèves, tarif de cinéma et prix du train). Un point supplémentaire était accordé pour chaque donnée correctement identifiée. Ensuite, un maximum de quatre points était accordé pour le calcul du coût proprement dit. Quelle que soit la stratégie adoptée par l'élève, un point a été attribué pour la présence de la démarche de recherche et un point pour celle du calcul du prix de la sortie par élève. Un point supplémentaire était accordé si ceux-ci étaient corrects.

7 Dans le cadre de la présente étude, le contrat proposé à l'enseignant de la classe contrôle est ambitieux, mais conforme aux exigences de la Fédération Wallonie-Bruxelles. En effet, bien qu'il soit clairement établi (Fagnant, Dupont \& Demonty, 2016; Mottier Lopez, 2012) que les enseignants rencontrent des difficultés à aider efficacement des élèves confrontés à des tâches complexes, le Ministère leur recommande de les utiliser sans fournir d'indications méthodologiques particulières. La situation rencontrée dans la classe contrôle nous semble dès lors traduire une certaine réalité de terrain. 


\section{Références}

Allal, L. (2007). Régulations des apprentissages: orientations conceptuelles pour la recherche et la pratique en éducation. In L. Allal \& L. Mottier Lopez (Éd.), Régulation des apprentissages en situation scolaire et en formation (pp. 7-23). Bruxelles: De Boeck.

Allal, L. (2011). Pedagogy, didactics and the co-regulation of learning: A perspective from the French-language world of educational research. Research Papers in Education, 26(3), 329-336.

Beckers, J. (2012). Introduction et mise en perspective théorique. In J. Beckers, J. Crinon \& G. Simons (Éd.), Approche par compétences et réduction des inégalités d'apprentissage entre élèves: de l'analyse des situations scolaires à la formation des enseignants (pp. 7-16). Bruxelles: De Boeck.

Buchs, C., Lehraus, K. \& Crahay, M. (2012). Coopération et apprentissage. In M. Crahay (Éd.), L'école peut-elle être juste et efficace? (pp. 421-454). Bruxelles: De Boeck.

Carette, V. (2007). L'évaluation au service de la gestion des paradoxes liés à la notion de compétence. Mesure et Évaluation en Éducation, 30(2), 49-71.

Chenu, F., Crahay, M. \& Lafontaine, D. (2014). Par-delà l'approche par compétences: quelle place réserver aux savoirs, à leur enseignement et à leur évaluation? Éducation \& Formation. e-302, 17-29.

Cobb, P., Zhao, Q. \& Visnovska, J. (2008). Learning from and adapting the theory of Realistic Mathematics Education. Éducation \& Didactique, 2(1), 105-124.

Communauté française (2008). Épreuve externe commune: CEB. Nombres et opérations (livret 1). Bruxelles: Ministère de la Communauté française, Administration générale de l'Enseignement et de la Recherche scientifique.

Communauté française (2008). Épreuve externe commune: CEB. Traitement de données (livret 5). Bruxelles: Ministère de la Communauté française, Administration générale de l'Enseignement et de la Recherche scientifique.

Communauté française (2011). Épreuve externe commune: CEB. Traitement de données et situations problèmes (livret 4). Bruxelles: Ministère de la Communauté française, Administration générale de l'Enseignement et de la Recherche scientifique.

Communauté française (2012). Épreuve externe commune: CEB. Traitement de données (livret 4). Bruxelles: Ministère de la Communauté française, Administration générale de l'Enseignement et de la Recherche scientifique.

Crahay, M. (2006). Dangers, incertitudes et incomplétude de la logique de compétence. Revue française de pédagogie, 154, 97-110.

Crahay, M. \& Detheux, M. (2005). L'évaluation des compétences, une entreprise impossible? (Résolution de problèmes complexes et maitrise de procédures mathématiques). Mesure et Évaluation en Education, 28(1), 57-78.

De Corte, E. \& Verschaffel. L. (2008). Apprendre et enseigner les mathématiques: un cadre conceptuel pour concevoir des environnements d'enseignement-apprentissage stimulants. In M. Crahay, L. Verschaffel, E. De Corte \& J. Grégoire (Éd.), Enseignement et apprentissage des mathématiques: que disent les recherches psychopédagogiques? (pp.25-54). Bruxelles: De Boeck Université.

De Corte, E., Verschaffel, L. \& Masui, C. (2004). The CLIA-model: A framework for designing powerful learning environments for thinking and problem solving. European Journal of Psychology of Education, 19(4), 365-384.

De Ketele, J.-M. (2010). Ne pas se tromper d'évaluation. Revue française de linguistique appliquée, 15(1), 25-37.

Demonty, I. \& Fagnant, A. (2014). Tâches complexes en mathématiques : difficultés des élèves et exploitations collectives en classe. Education et francophonie, 42(2), 173-189.

Eurydice. (2012). Developing Key Competences at School in Europe: Challenges and Opportunities for Policy (Eurydice Report). Luxembourg: Publications Office of the European Union. 
Fagnant, A., Dupont, V. \& Demonty, I. (2016). Régulation interactive et résolution de tâches complexes en mathématiques. In L. Mottiez Lopez \& W. Tessaro (Éd.), Les processus de jugement dans des pratiques d'évaluation des apprentissages (pp. 229-251). Berne: Peter Lang.

Fagnant, A. \& Vlassis, J. (2013). Schematic representations in arithmetical problem solving: Analysis of their impact on grade 4 students. Educational Studies in Mathematics, 84, 149-168.

Focant, J. \& Grégoire, J. (2008). Les stratégies d'autorégulation cognitive: une aide à la résolution de problèmes arithmétiques. In M. Crahay, L. Verschaffel, E. De Corte \& J. Grégoire (Éd.), Enseignement et apprentissage des mathématiques: que disent les recherches psychopédagogiques? (pp. 201-221). Bruxelles: De Boeck.

Ginsburg, A., Cooke, G., Leinwand, S., \& Pollock, E. (2005). Reassessing U.S. international mathematics performance: New findings from 2003 TIMSS and PISA. Washington, D.C.: American institute for Research.

Jonnaert, P. (2009). Compétences et socio-constructivisme: un cadre théorique (2e édition). Bruxelles: De Boeck.

Julo, J. (1995). Représentation des problèmes et réussite en mathématiques: un apport de la psychologie cognitive à l'enseignement. Rennes: Presses universitaires.

Marcoux, G. (2014). Résolution de problèmes arithmétiques dans le cadre d'une approche par compétences: ordre des tâches et parts d'influence de quelques facteurs cognitifs et motivationnels. Les cahiers des Sciences de l'Éducation, 36, 67-114.

Mevarech, Z. R. \& Kramarski, B. (2014). Critical maths for innovative societies: The role of metacognitive pedagogies. Paris: OECD publishing.

Montague, M. (2007). Self-regulation and mathematics instruction. Learning Disabilities Research \& Practice, 22(1), 75-83.

Mottier Lopez, L. (2012). La régulation des apprentissages en classe. Bruxelles: De Boeck.

Özoy, G. \& Ataman, A. (2009). The effect of metacognitive strategy training on mathematical problem solving achievement. International Electronic Journal of Elementary Education, 1(2), 67-82.

Perels, F., Gürtler, T. \& Schmitz, B. (2005). Training of self-regulatory and problem-solving competence. Learning and Instruction, 15, 123-139.

Perrenoud, P. (1998a). L'évaluation des élèves. De la fabrication de l'excellence à la régulation des apprentissages: entre deux logiques. Bruxelles: De Boeck.

Perrenoud, P. (1998b). From Formative Evaluation to a Controlled Regulation of Learning Processe: Towards a wider conceptual field. Assessment in Education, 5(1), 85-102.

Rey, B., Carette, V., Defrance, A. \& Kahn, S. (2006). Les compétences à l'école: apprentissage et évaluation (2 édition). Bruxelles: De Boeck.

Van Dooren, W., Verschaffel, L., Greer, B., De Bock, D., Crahay, M. (2010). La modélisation des problèmes mathématiques. In: Crahay M., Dutrévis M. (Éd.), Psychologie des apprentissages scolaires (2e édition, chap. 8, pp. 199-220). Bruxelles: De Boeck.

Verschaffel, L. \& De Corte, E. (2008). La modélisation et la résolution des problèmes d'application: de l'analyse à l'utilisation efficace. In M. Crahay, L. Verschaffel, E. De Corte \& J. Grégoire (Éd.), Enseignement et apprentissage des mathématiques: que disent les recherches psychopédagogiques? (2e édition, pp. 153-176). Bruxelles: De Boeck.

Verschaffel, L., De Corte, E., Lasure, S., Van Vaerenbergh, G., Bogaerts, H. \& Ratinckx, E. (1999). Learning to solve mathematical application problems: A design experiment with fifth graders. Mathematical Learning and Thinking, 1, 195-299.

Mots-clés: Tâches complexes de mathématiques, modèle d'environnements d'enseignement/apprentissage stimulants, résolution de problèmes, métacognition, co-régulation. 


\section{Wie kann mathematisches Problemlösen durch co-regulative und co-konstruktive Unterrichtsmethoden in der Grundschule gefördert werden?}

\section{Zusammenfassung}

Die vorliegende Studie testet eine Unterrichtsmethode, die komplexe mathematische Aufgaben enthält, mit Grundschülerinnen und -schülern der 6. Klasse (Schüler im Alter von 11-12 Jahren) im französischsprachigen Teil Belgiens. Diese Unterrichtsmethode basiert auf den Grundwerten eines stimulierenden Lernumfelds (De Corte \& Verschaffel, 2008), sowie auf den Studien von Allal (2007, 2011) und Mottier Lopez (2012) bezüglich des Einsatzes der interaktiven und der co-regulierten Unterrichtsmethode. Die quasi-experimentelle Studie verwendet qualitative Analysemethoden. Die Ergebnisse zeigen die Wirksamkeit der Unterrichtsmethode; insbesondere auch bei schwächeren Schülern, sofern eine adäquate Struktur besteht. Vergleiche zwischen Versuchs- und Kontrollklassen unterstreichen die zentrale Rolle der Lehrperson zur Förderung der mathematischen Problemlösefähigkeiten der Schülerinnen und Schüler.

Schlagworte: Komplexe mathematische Aufgaben, stimulierenden Lernumfelds, mathematisches Problemlösen, metakognition, co-regulative.

\section{Favorire la co-regolazione e la co-costruzione di un processo efficace di risoluzione di problemi matematici al termine della scuola primaria}

\section{Riassunto}

Il presente studio sta testando un dispositivo d'insegnamento di compiti complessi di matematica presso allievi della sesta primaria (11-12 anni) nel Belgio francofono. Lo studio si basa sui principi costitutivi del modello degli ambienti d'insegnamento/apprendimento stimolanti (De Corte \& Verschaffel, 2008), così come sui lavori di Allal $(2007,2011)$ e Mottier Lopez (2012) relativi alle regolazioni interattive e alla co-regolazione. Lo studio combina un dispositivo quasi-sperimentale e analisi qualitative. I risultati mostrano l'efficacia del dispositivo sviluppato, anche presso gli alunni più deboli, a patto di fornire loro un sostegno adeguato. I paragoni tra classi sperimentali e di controllo sottolineano il ruolo centrale dell'insegnante per fare evolvere il ragionamento matematico degli alunni.

Parole chiave: Compiti complessi di matematica, ambienti d'insegnamento/ apprendimento stimolanti, risoluzione di problemi, metacognizione, co-regolazione. 


\section{How teachers can promote co-regulation and co-construction of a skilled approach in mathematical problem solving at the end of primary education}

\section{Summary}

The present study experiments the impact of a teaching device centered on mathematical complex tasks among primary education pupils (grade 6) in French-speaking Belgium. This device is based on the characteristics of the powerful learning environment (De Corte \& Verschaffel, 2008) on the one hand, and on Allal $(2007,2011)$ and Mottier Lopez (2012) researches about interactive regulations and coregulation on the other hand. The study combines a quasi-experimental design with qualitative data analysis. The results show that the teaching device leads to an improvement of pupils skills (or an increase of performance), including among low achievers, if an appropriate scaffolding is provided. Comparisons between experimental and control groups highlight the importance of the teacher' role in order to improve pupils mathematical reasoning.

Keywords: Mathematical complex tasks, powerful learning environment, problem solving, metacognition, co-regulation. 
